\title{
Sampling the host response to SARS-CoV-2 in hospitals under siege
}

To the Editor - Severe acute respiratory syndrome coronavirus 2 (SARS-CoV-2) and the resulting coronavirus disease 2019 (COVID-19) have stretched healthcare systems thin across the world. During a COVID-19 surge, local academic medical centers must both care for the ill and perform research to better understand the pathogenicity of this disease. In this Correspondence, we provide an account of a 49-day period during which we built a COVID-19 research biobank with samples from patients hospitalized in a large New York City health system. For like-minded colleagues in future hot spots, this is not necessarily a model to follow, but rather a live-and-learn memoir of our actions and mistakes under uniquely strenuous conditions.

Our mandate was to design a sample-collection protocol to serve as the backbone for COVID-19 research by yielding high-quality source material for sensitive molecular assays (e.g., RNA sequencing and CyTOF) that cannot be performed on clinical discards. We began by assembling a volunteer army of over 100 clinical and research staff members who were readily available for redeployment because their usual research or clinical assignments had been shut down by the COVID-19 surge. Teams were organized for screening electronic medical records, obtaining informed consent, assembling collection kits, performing nurse outreach, transporting specimens, processing samples, and biobanking (Fig. 1a).

Each morning the screening team reviewed the electronic medical records of all patients hospitalized in the previous 24 hours and, with input from team physicians, selected up to 30 for biobank enrollment. The inclusion criteria were broad, including both patients with COVID-19 and control participants, while the exclusion criteria were minimal. The assembly team was responsible for packing Vacutainers into biohazard bags ('kits'). 'Running' team members brought empty kits to the units and left them in pouches taped to the hospital-room doors of study participants. The kits were filled by nurses or phlebotomists when blood draws were done as part of clinical care, to minimize exposure of the clinical staff to SARS-CoV-2.

Outreach was essential to explain to a

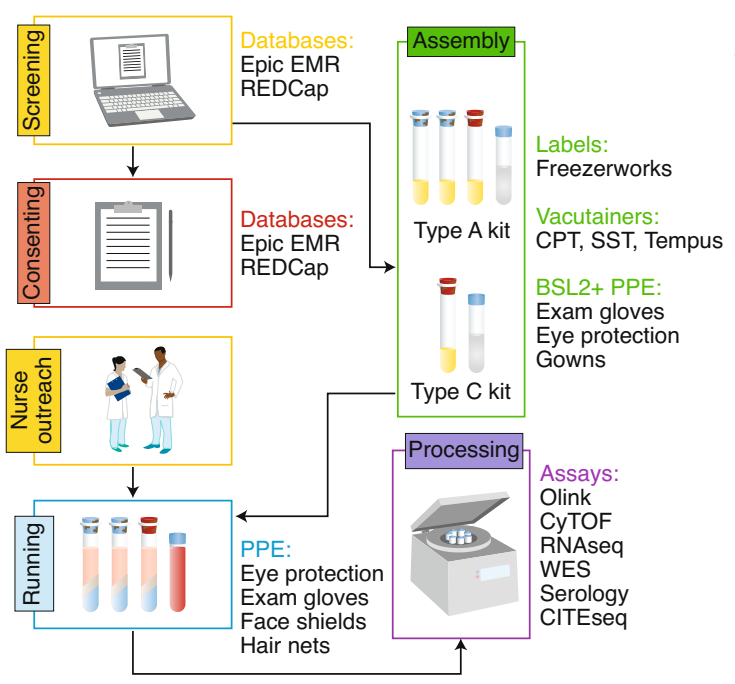

b

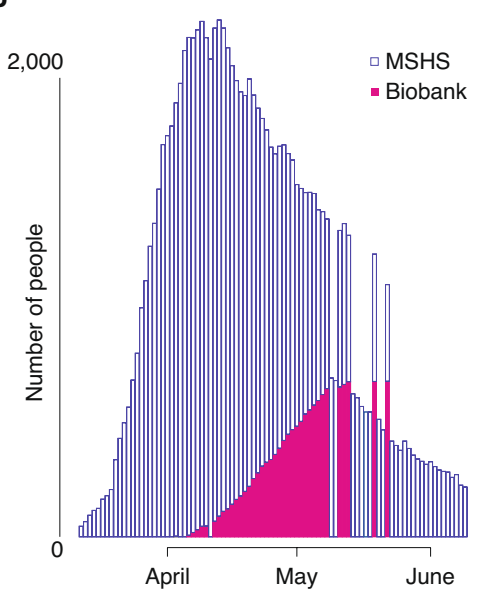

Fig. 1 | Mount Sinai COVID-19 biobank workflow and collection timeline. a, Mount Sinai COVID-19 biobank workflow. The workflow involved six teams of research personnel. First, the screening team identified eligible patients and notified the assembly and consenting teams. The assembly team then created study kits while the nurse outreach team engaged with the clinical staff. Once kits were assembled, the running team distributed kits to and collected kits from the clinical staff. Finally, the running team transported filled kits to the processing team. EMR, electronic medical records; PPE, personal protective equipment; CPT, cell-preparation tube; SST, serum-separator tube; CyTOF, cytometry by time of flight; RNAseq, RNA sequencing; WES, whole-exome sequencing; CITEseq, cellular indexing of transcriptomes and epitopes by sequencing. b, Timeline of Mount Sinai COVID-19 biobank collection. Bars indicate the number of people (vertical axis) hospitalized with COVID-19 in the Mount Sinai Health System (MSHS) or enrolled in the biobank (cumulative) at a given time point (horizontal axis).

clinical staff the role of biobank collections in combating COVID-19. Toward this end, runners participated in daily 'nurse huddles' on the units, and it was common for running team leads to receive calls from clinical staff members in the middle of the night seeking clarity on study procedures before they filled the kits. Runners retrieved filled kits when they dropped empty kits off for the subsequent draw. They delivered hundreds of kits daily to the laboratory, whereupon the sample-processing team carried out an involved procedure that ended with the banking of serum, plasma, whole blood, and peripheral blood mononuclear cells. Processing had to be performed in biosafety level 2 plus (BSL-2+) facilities, but as our operation grew, there were not enough such facilities to handle the volume. We overcame this by converting tissue-culture rooms throughout campus to
BSL-2+ specifications. Screening, collection, and processing occurred 7 days a week during the 49-day period.

So that samples and data could be used and shared broadly, informed consent was strongly desired. On the first day of enrollment, however, we realized it was impractical. In part, this was the result of necessary infection-control policies (e.g., strict limits on entry to COVID19 units). It was also due to the illness itself. Not only are hospitalized patients with COVID-19 dyspneic, they are also under intense psychological duress with a mysterious condition, with visitors barred and human contact limited to clinical staff members wearing masks and face shields. To facilitate mission-critical research in these circumstances, the Institutional Review Board allowed sample collection before informed consent was obtained. Participants 
were made aware of this via documents provided during hospital registration and were given instructions for opting out. Consenting team members attempted to contact hospitalized participants via the hospital-room phone, but most were unreachable throughout admission. Obtaining consent was next attempted by phone following discharge or, as was too often the case, after death (through legally authorized representatives). The majority of people from whom samples were obtained have provided consent at the time of this writing.

The work was done in a fog-of-war environment. Constant obstacles created considerable room for error but also inspired creative solutions. Daily team meetings were held to maintain morale. Several staff members with no previous clinical experience witnessed death first-hand. Many routinely worked 20-hour days with long stretches in personal protective equipment that led to overheating and dehydration. Permeating every moment was the fear of infection. Only two staff members developed COVID-19, a low rate we attribute to strict use of personal protective equipment and adherence to hand hygiene. When we reached our target sample size of 500, our collective impulse was not to relent but to push full steam ahead. Conducting this research was therapeutic for the clinicians and scientists involved, providing a means of fulfilling their deep sense of duty to the community. In total, over 700 participants were accrued in 49 days, with over 10,000 Vacutainers filled and over 50,000 aliquots banked (Fig. 1b).

Most team members have since returned to their previous assignments, pledging to reassemble if a second wave strikes New York City. Until then, our energy will go toward extracting actionable knowledge from molecular data generated from these samples. As other regions encounter what we encountered in our catchment area, we encourage similar biobanking efforts. The keys to achieving our goal were rigor, stamina, and teamwork.
Alexander W. Charney ${ }^{1 凶}$

Nicole W. Simons ${ }^{1}$, Konstantinos Mouskas',

Lauren Lepow', Esther Cheng',

Jessica Le Berichel' ${ }^{1}$, Christie Chang (D)',

Robert Marvin', Diane Marie Del Valle',

Sharlene Calorossi', Alona Lansky',

Laura Walker', Manishkumar Patel', Hui Xie',

Nancy Yi', Alex Yu', Gurpawan Kang (D)',

Anthony Mendoza', Lora E. Liharska',

Emily Moya', Matthew Hartnett',

Sandra Hatem ${ }^{1}$, Lillian Wilkins ${ }^{1}$,

Melody Eaton', Hajra Jamal', Kevin Tuballes',

Steven T. Chen ${ }^{1}$, Alexandra Tabachnikova',

Jonathan Chung', Jocelyn Harris',

Craig Batchelor', Jose Lacunza',

Mahlet Yishak ${ }^{1}$, Kimberly Argueta',

Neha Karekar', Brian Lee', Geoffrey Kelly',

Daniel Geanon', Diana Handler', John Leech',

Hiyab Stefanos', Travis Dawson', leisha Scott',

Nancy Francoeur', Jessica S. Johnson?',

Akhil Vaid', Benjamin S. Glicksberg (iD)',

Girish N. Nadkarni', Eric E. Schadt',

Bruce D. Gelb', Adeeb Rahman',

Robert Sebra', Glenn Martin', The

Mount Sinai COVID-19 Biobank Team*,

Thomas Marron', Noam Beckmann ${ }^{1,2}$,

Seunghee Kim-Schulze ${ }^{1,2}$, Sacha Gnjatic (D) 1,2 and Miriam Merad ${ }^{1 凶}$

${ }^{1}$ Icahn School of Medicine at Mount Sinai,

New York, NY, USA. ${ }^{2}$ These authors contributed

equally: Noam Beckmann, Seunghee Kim-Schulze,

Sacha Gnjatic. ${ }^{*} A$ list of authors and their affiliations

appears at the end of the paper.

凶e-mail: alexander.charney@mssm.edu;

miriam.merad@mssm.edu

Published online: 27 July 2020

https://doi.org/10.1038/s41591-020-1004-3

Acknowledgements

This work was accomplished by a redeployed workforce supported by the following centers, programs, departments, and institutes within the Icahn School of Medicine at Mount Sinai: Human Immune Monitoring Center; Mount Sinai COVID-19 Informatics Center; Program for the Protection of Human Subjects; Department of Psychiatry; Department of Genetics and Genomic Sciences; Department of Medicine; Department of Oncological Sciences; Department of Pediatrics; The Precision Immunology Institute; Tisch Cancer Institute; Icahn Institute for Data Science and Genomic Technology; Friedman Brain Institute; Charles Bronfman Institute of Personalized Medicine; Hasso Plattner Institute for Digital
Health; Mindich Child Health and Development Institute; and Black Family Stem Cell Institute.

Competing interests

The authors declare no competing interests.

The Mount Sinai COVID-19 Biobank Team

Charuta Agashe', Priyal Agrawal', Alara Akyatan', Kasey Alesso-Carra', Eziwoma Alibo', Kelvin Alvarez', Angelo Amabile', Steven Ascolillo', Rasheed Bailey', Priya Begani', Paloma Bravo Correra', Stacey-Ann Brown', Mark Buckup', Larissa Burka', Lena Cambron', Gina Carrara', Serena Chang', Jonathan Chien', Mashkura Chowdhury', Cansu Cimen Bozkus', Phillip Comella', Dana Cosgrove',

Francesca Cossarini', Liam Cotter', Arpit Dave', Bheesham Dayal', Maxime Dhainaut', Rebecca Dornfeld', Katie Dul', Nissan Eber', Cordelia Elaiho', Frank Fabris', Jeremiah Faith', Dominique Falci', Susie Feng', Brian Fennessy', Marie Fernandes', Sandeep Gangadharan', Joanna Grabowska', Gavin Gyimesi', Maha Hamdani', Manon Herbinet ${ }^{1}$, Elva Herrera', Arielle Hochman', Gabriel E. Hoffman', Jaime Hook', Laila Horta', Etienne Humblin', Subha Karim', Jessica Kim', Dannielle Lebovitch', Grace Lee', Gyu Ho Lee', Jacky Lee', Mike Leventhal', Katherine Lindblad', Alexandra Livanos', Rosalie Machado', Zafar Mahmood', Kelcey Mar', Shrisha Maskey', Paul Matthews', Katherine Meckel', Saurabh Mehandru', Cynthia Mercedes', Dara Meyer', Gurkan Mollaoglu', Sarah Morris', Kai Nie1, Marjorie Nisenholtz', George Ofori-Amanfo', Kenan Onel', Merouane Ounadjela', Vishwendra Patel', Cassandra Pruitt', Shivani Rathi', Jamie Redes', Ivan Reyes-Torres', Alcina Rodrigues', Alfonso Rodriguez', Vladimir Roudko', Evelyn Ruiz', Pearl Scalzo', Pedro Silva', Alessandra Soares Schanoski', Meghan Straw', Sasha Tabachnikova', Collin Teague', Bhaskar Upadhyaya', Verena Van Der Heide', Natalie Vaninov', Daniel Wacker', Hadley Walsh',

C. Matthias Wilk', Jessica Wilson', Karen M. Wilson', Li Xue',

Naa-akomaah Yeboah', Sabina Young', Nina Zaks ${ }^{1}$ and Renyuan Zha' 